\section{Cerebral dominance for the perception of rhythm}

\author{
ANDREW H. GREGORY, JAMES C. HARRIMAN* \\ and LORNA D. ROBERTS \\ University of Manchester, Manchester M13 9PL, England
}

Five right-handed Ss listened to stimuli presented alternately to each ear at a rate of $2 / \mathrm{sec}$ and adjusted the precise timing of the stimulus to one ear so that the rhythm appeared completely regular. The mean errors in this setting were measured for a control condition with loud stimuli, and for an experimental condition with near-threshold stimuli, and sinusoidally modulated white noise in the ear opposite to the stimulus. The results show a significant difference of about 4 msec between the two conditions, the stimulus to the right ear being apparently delayed in the experimental condition relative to the stimulus to the left ear. This suggests that the right cerebral hemisphere is involved in the perception of rhythmic stimuli.

In normal right-handed Ss, the left cerebral hemisphere appears to be dominant for speech, while some nonverbal auditory functions appear to be specialized in the right hemisphere. It is not, however, clear if the perception of rhythm tends to be localized mainly in one hemisphere. Milner (1962), using the Seashore Measures of Musical Talents, found that although performance on the tests of timbre and tonal memory was impaired by right temporal lobectomy, the rhythm test was not significantly impaired by either left or right temporal lobectomy.

Tsunoda $(1966,1968)$ has studied rhythm production in normal Ss. His Ss tapped rhythms while listening through headphones to pure tones triggered by their taps. The auditory feedback to one ear was then delayed and increased in intensity until it produced disruption of the tapping response. He reported that, using pure tones, delay on the left ear is relatively more disruptive than it is on the right, thus suggesting a right hemisphere preference for rhythm, since the contralateral auditory pathway is known to be more effective than the ipsilateral pathway (Rosenzweig, 1951).

With normal right-handed Ss, Kimura (1964) found a significant right-ear advantage for reporting dichotically presented digits and a significant left-ear advantage for dichotic melodies. Since Rosenzweig (1951) has shown from electrophysiological recording on cats that more units respond in the auditory cortex contralateral to the stimulated ear, Kimura's experiments support the idea of a degree of specialization for speech in the left

\footnotetext{
* Present address: Psychology Department, University of Bristol, England.
}

hemisphere and for at least some aspects of musical perception in the right hemisphere.

On the other hand, Efron (1963) provided evidence that the temporal discrimination of simultaneity and order is performed in the left hemisphere by right-handed Ss. Since rhythm perception basically involves temporal discrimination, one might expect the same hemisphere to be preferred for both judgments. The present study is designed to determine which cerebral hemisphere is involved in the perception of rhythm by using a alternately to each hemisphere and measuring the mean error made by $\mathrm{Ss}$ in setting it to apparent regularity.

$$
\text { METHOD }
$$

When a near-threshold stimulus is applied to one ear at the same time as rhythmical stimulus which is presented varied in precise timing by the $S$. masking noise is to the other ear, then, as the contralateral pathway is more effective, the signal/noise ratio will be relatively much higher in the hemisphere contralateral to the ear receiving the signal. Such a stimulus presented alternately to each ear will thus go alternately to each hemisphere. If the perception of rhythm tends to be specialized in one hemisphere, every alternate stimulus will have to be relayed across from the other hemisphere, presumably through the corpus callosum, and the extra delay involved should result in a constant error in setting the repeated stimulus to be regular. From the direction of the error measured in the experiment, one can conclude which hemisphere is being used for the judgment. To compensate for any slight difference in the sensitivity of the ears for any one $S$, this experimental condition was compared with a control condition using a relatively intense stimulus alternating between the ears, relayed to both hemispheres each time it occurs.

The stimuli and masking noise were obtained from an audiometer (Amplivox, Model 82) feeding into a TDH-39 headset, and the timing was controlled by external logic circuits. The stimuli were $1-\mathrm{kHz}$ pure tones of $70-\mathrm{msec}$ duration, with a rise and fall time of about $7 \mathrm{msec}$, and were presented repeatedly, alternating between ears. On one ear, the stimuli were presented precisely every 1,000 msec. On the other ear, the timing of the stimulus could be adjusted by the $S$ over a range of 430 to $570 \mathrm{msec}$ after the stimulus on the first ear. The $S$ was asked to adjust the timing of the variable stimulus so that

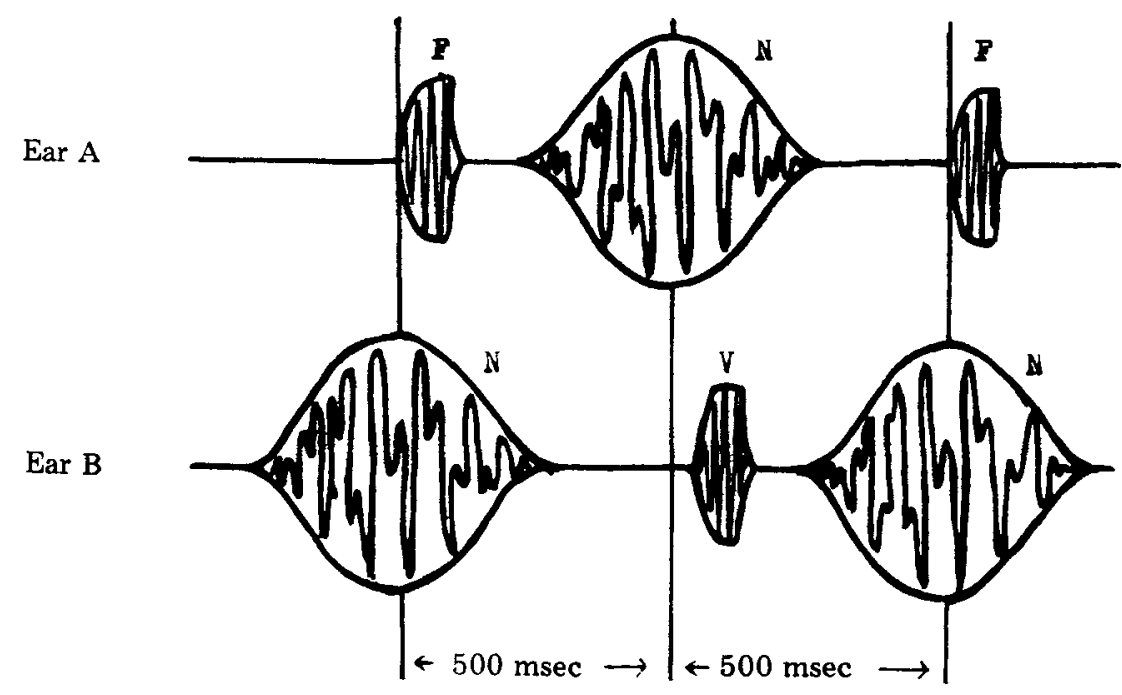

Fig. 1. The sequence of stimulation at the two ears. The modulated noise, $N$, and the fixed stimulus, $F$, appear at fixed intervals; the other stimulus, $V$, is 
Table 1

Mean and Standard Deviation of the Error in Milliseconds in Setting the Timing of the Variable Stimulus

\begin{tabular}{|c|c|c|c|c|c|}
\hline \multirow[b]{2}{*}{$\mathrm{S}$} & \multirow[b]{2}{*}{$N$} & \multicolumn{2}{|c|}{ Control Condition } & \multicolumn{2}{|c|}{ Experimental Condition } \\
\hline & & MI & SD & MI & $\mathrm{SD}$ \\
\hline 1 & 48 & 6.56 & 12.09 & 0.42 & 10.00 \\
\hline 2 & 48 & --4.83 & 11.51 & -5.39 & 14.64 \\
\hline 3 & 48 & -2.76 & 12.70 & -1.51 & 15.23 \\
\hline 4 & 96 & 5.71 & 13.56 & -2.41 & 14.17 \\
\hline 5 & 96 & 4.49 & 9.87 & -1.28 & 13.95 \\
\hline \multicolumn{2}{|c|}{$\begin{array}{l}\text { Orerall Mean } \\
\text { (Unweighted) }\end{array}$} & 1.83 & & -2.03 & \\
\hline
\end{tabular}

the continuous rhythm appeared completely regular. He was allowed as long as he wished to make this adjustment, but a typical time would be about $1 \mathrm{~min}$. The time set by the $S$ was measured to an accuracy of $0.1 \mathrm{msec}$ and expressed as a difference from the ideal time of $500 \mathrm{msec}$.

In the control condition, the stimulus intensity was $60 \mathrm{~dB}$ SPL. In the experimental condition, the stimulus intensity was set as low as possible consistent with making the judgment (typically $25 \mathrm{~dB}$ ), and sinusoidally modulated masking noise was presented to the opposite ear from the stimulus. This condition is illustrated in Fig. 1, where it can be seen that the masking noise $(N)$ also alternated between the ears every $1 / 2 \mathrm{sec}$. The masking noise was fixed in time in each ear, in synchrony with the fixed stimulus ( $F$ ), but the gradual rise and fall ensured that no cue was given as to the correct timing of the variable stimulus (V). The masking noise was narrow-band white noise with a bandwidth of about $\pm 50 \mathrm{~Hz}$ centered on $1 \mathrm{kHz}$ and thus masked the stimulus effectively without causing discomfort. The maximum noise intensity was $50 \mathrm{~dB}$, and the modulation was produced by a Cowan modulator circuit driven by a waveform generator synchronized with the timing control logic.

Five Ss were used, all being right-handed as measured by the Harris tests of lateral dominance. For each $\mathrm{S}$, the threshold for each ear was determined using a $1-\mathrm{kHz}$ tone, and in no case were there any differences between the ears of more than $5 \mathrm{~dB}$.

After a series of training trials with the control condition, readings were taken in blocks of 16 trials of either the experimental or the control condition on any one occasion. The headset was reversed every eight trials, and every four trials the fixed and variable stimuli were changed to the opposite ears. Readings were made in a quiet dark room. The control for the timing of the variable stimulus was a circular knob attached to a potentiometer with no stops at either end. There were thus no visual or kinesthetic cues as to its position, and, as an additional precaution, the relation between the direction of controi and its effect on the time was reversed on half the trials. The order of all conditions was randomized throughout the experiment.

$$
\text { RESULTS }
$$

Before calculating the means, the results of individual trials were all converted to the form of the difference from the ideal setting of $500 \mathrm{msec}$ for the condition where the reference stimulus goes to the left ear and the variable to the right. A negative value thus corresponds to a setting of the variable time to less than $500 \mathrm{msec}$; this implies that the variable stimulus, on the right ear, is being delayed in the brain relative to the fixed stimulus. In the experimental condition, this would suggest that the stimulus reaching the left hemisphere is delayed relative to that reaching the right hemisphere and thus probably hemisphere for the judgment to be made.

Table 1 shows the mean and standard deviation of the error in settings of the variable stimulus of each of the five Ss under the two conditions, together with the unweighted overall mean of all the Ss. A two-way analysis of variance showed a just-significant $S s$ by Conditions interaction $(\mathrm{F}=2.87, \mathrm{df}=4,662$, $p<.05$ ), but the two main variables were both very highly significant and so can be examined separately. Significant differences were found between Ss $(F=7.52, \quad d f=4,662$, $\mathrm{p}<.001)$ and between the two conditions $(F=22.64, \quad \mathrm{df}=1,662$, $p<.001)$. The overall mean of the five has to be transmitted to the right
Ss in the experimental condition was $-2.03 \mathrm{msec}$, and expressed relative to the control condition, this difference becomes $-3.86 \mathrm{msec}$.

\section{DISCUSSION}

The difference between the two conditions is clearly significant. As the mean time set in the experimental condition is negative relative to the control condition, this would suggest, as described above, that the right cerebral hemisphere is used for rhythm perception.

The differences between individual Ss in the control condition may be due to slight differences in the sensitivity of the two ears. This would result in alternate stimuli being of apparently different intensities. Fraisse (1956) has shown that this distorts the apparent time intervals between pairs of stimuli.

It is perhaps still slightly surprising that the mean error over all $\mathrm{Ss}$ is about $+2 \mathrm{msec}$ in the control condition. A possible explanation is that the nerve pathway from the ear to the ipsilateral hemisphere has fewer synapses, and therefore conducts more quickly, than that to the contralateral hemisphere. If the right hemisphere is used for rhythm perception then in the control condition, it would receive the right-ear stimulus more quickly, by the ipsilateral pathway, than the left-ear stimulus. This may be contrasted with the experimental condition where the iosilateral pathway is suppressed by masking noise and the left-ear stimulus is received more quickly.

\section{REFERENCES}

EFRON, R. The effect of handedness on the perception of simultaneity and temporal order. Brain, 1963, 86, 261-284.

FRAISSE, P. Les structures rythmiques. Louvain: Publications Universitaires de Louvain, 1956

KIMURA, D. Left-right differences in the perception of melodies. Quarterly Journal of Experimental Psychology, 1964, 16. 355-358.

MILNER. B. Laterality effects in audition. In V. B. Mountcastle (Ed.). Interhemispheric relations and cerebral dominance. Baltimore: Johns Hopkins Press, 1962. Pp. 177-195.

ROSENZWEIG, M. R. Representations of the two ears at the auditory cortex. American Journal of Physiology, 1951, $167,147-158$

TSUNODA, T. Tsunoda's method: A new objective testing method available for the orientation of the dominant cerebral hemisphere towards various sounds and its clinical use. Indian Journal of Otology, $1966,8,78-88$

TSUNODA, T An audiological approach to speech disorders due to cortical lesion. Practica Otologica Kyoto, 1968, 61, Supplement 92-96. 